\section{Klebsiella pneumoniae carbapenemase production among $K$. pneumoniae isolates and its concern on antibiotic susceptibility}

\author{
Azam Shahi,, 1,2 Alka Hasani, 1,2 \\ Mohammad Ahangarzadeh Rezaee, ${ }^{1,2}$ \\ Mohammad Asghari Jafarabadi, ${ }^{3}$ \\ Akbar Hasani, ${ }^{4}$ \\ Hossein Samadi Kafil, ${ }^{1,2}$ \\ Mohammad Yosef Memar, ${ }^{2}$ \\ Elgar Soltani ${ }^{1,2}$ \\ ${ }^{1}$ Immunology Research Center; \\ 2Department of Medical Microbiology, \\ Faculty of Medicine; ${ }^{3}$ Department of \\ Statistics and Epidemiology; \\ ${ }^{4}$ Drug Applied Research Center and \\ Department of Clinical Biochemistry \\ and Laboratory Sciences, Faculty of \\ Medicine, Tabriz University of Medical \\ Sciences, Tabriz, Iran
}

\begin{abstract}
The emergence of Klebsiella pneumoniae carbapenemase (KPC) resistance has led to the countdown of activity of carbapenems, which were considered as drugs of last resort for infections caused by Enterobacteriaceae. The aims of the present study were the detection of KPC-production among $K$. pneumoniae isolates, select the appropriate method for its detection and assess the consequence of KPC production on the antibiotics susceptibility. One hundred and four non-duplicated $K$. pneumoniae isolates were collected from University teaching hospitals of Tabriz, Iran. The disk diffusion, E-test, and Modified Hodge test were performed for the determination of antibiotic susceptibility pattern, Minimum Inhibitory Concentrations (MICs) determination and the production of carbapenemase, respectively. $B l a_{K P C-2}$ gene was detected by using PCR. High levels of resistance were observed towards co-trimoxazole (69.2\%), followed by cefazolin $(66.3 \%)$, ceftriaxone $(65.4 \%)$, ofloxacin and ciprofloxacin $(54.8 \%)$, gentamicin $(50 \%)$, and amikacin (39.4\%). According to the disk diffusion method, the frequency of imipenem and meropenem resistance was $31.7 \%$ and $32.7 \%$, respectively. Colistin was the most effective antibiotic among panels of antibiotics tested. Imipenem MICs range, $\mathrm{MIC}_{50}$ and $\mathrm{MIC}_{90}$ were $0.19-32 \mu \mathrm{g} / \mathrm{ml}, 4 \mu \mathrm{g} / \mathrm{ml}$, and $16 \mu \mathrm{g} / \mathrm{ml}$, respectively. Modified Hodge test was positive in $24(63.2 \%)$ isolate however,
\end{abstract}

$b l a_{K P C-2}$ gene was detected in $8(21.1 \%)$ carbapenem-resistant isolates. Results of the present study revealed a high rate of carbapenem-resistance in $K$. pneumoniae by phenotypic method, however the presence of one of the molecular, namely $b l a_{K P C-2}$ was not found as predominant cause. Therefore, their reliable detection should be the first priority to combat the infections. Being a simple test, the imipenem disk diffusion could be considered as an appropriate method for the detection of carbapenem-resistant isolates in the routine diagnosis.

\section{Introduction}

Klebsiella pneumoniae is one of the leading etiology of nosocomial infections, afflicting frequently intensive care unit (ICUs) patients and those who are immunocompromised. The organism has gained much attention due to the strikingly increased emergence of multi-drug resistant (MDR) $K$. pneumoniae strains. ${ }^{1-3}$ Till last decade carbapenems possessed most consistent in-vitro activity especially towards extended-spectrum-lactamase (ESBL)-producing $K$. pneumoniae, nevertheless resistance against these antibiotics has increased and represents a significant threat in the management of MDR isolates. ${ }^{4}$ Carbapenemresistant Enterobacteriaceae isolates are resistant to almost all classes of antimicrobials, and infections are associated with extremely high rates of morbidity and mortality. ${ }^{5,6} \mathrm{~K}$. pneumoniae constitutes $92 \%$ of all carbapenem-resistant Enterobacteriaceae in the United States and Klebsiella pneumoniae carbapenemase (KPC) is one of the most predominant mechanisms of resistance to carbapenems. ${ }^{7}$ Antimicrobial resistance has become one of the most serious public health concerns worldwide. It is a global rather than a local issue, as antimicrobial resistance can spread between countries or continents. ${ }^{8}$ Based on the global spread of KPC-producing bacteria and limitations of the current treatments, the implementation of prevention and control measures has become extremely important. ${ }^{9,10}$ We conducted a study for the detection of KPC-producing $K$. pneumoniae isolates, emphasizing their prevalence, antibiotics susceptibility and selection of the appropriate recognition method in our setting.

\section{Materials and Methods}

\section{Sample collection and $K$. pneumoni- ae isolation}

One hundred and four $K$. pneumoniae
Correspondence: Alka Hasani, Immunology Research Center and Department of Medical Microbiology, Faculty of Medicine, Tabriz University of Medical Sciences, Tabriz, Iran. Tel.: 0098.413.3364661.

E-mail: dr.alkahasani@gmail.com

Key words: Klebsiella pneumoniae; Bla $a_{K P C 2}$; Carbapenemase; Modified Hodge test.

Contributions: AS developed the methodology, carried out the experiment and wrote the manuscript with input from all authors; $\mathrm{AlH}$ conceived and planned the experiments, supervised development of work, helped in data interpretation and writing manuscript and its evaluation; MAR conceived and planned the experiments; MAJ contributed to the interpretation of the results; AkH helped to evaluate and edit the manuscript; HSK assembled input data and performed the analysis; MYM worked out almost all of the technical details of each experiment with AS and AH; ES processed the experimental data. All authors discussed the results and contributed to the final manuscript.

Conflict of interest: the authors declare no potential conflict of interest.

Funding: This work was financially supported by the Immunology Research Center, Tabriz University of Medical Sciences, Tabriz, Iran. The funders had no role in study design, data collection and interpretation.

Received for publication: 18 January 2018. Revision received: 1 September 2018.

Accepted for publication: 2 September 2018

This work is licensed under a Creative Commons Attribution NonCommercial 4.0 License (CC BY-NC 4.0).

CCopyright A. Shahi et al., 2019

Licensee PAGEPress, Italy

Microbiology Research 2019; 10:7587

doi:10.4081/mr.2019.7587

isolates were collected from a general University teaching hospital, Tabriz, Iran. Blood, urine and wounds represented the source of the specimens. Gram staining and standard biochemical tests including reaction in triple sugar iron agar, motility, indole production and citrate utilization, were performed for the identification of isolates in the Department of Microbiology, Tabriz University of Medical Sciences, Tabriz.

\section{Antimicrobial susceptibility pattern}

Antibiotic susceptibility pattern of isolates was determined by performing KirbyBauer method (disk diffusion test) using Muller-Hinton agar according to guidelines 
of the CLSI. ${ }^{11}$ All isolates were checked for their susceptibility or resistance towards Trimethoprim/sulfamethoxazole or co-trimoxazole (TS) $(1.25 / 23.75 \mu \mathrm{g})$, ceftriaxone (CRO) $(30 \mu \mathrm{g})$, ceftazidime (CAZ) $(30 \mu \mathrm{g})$, amikacin $(\mathrm{AK})(30 \mu \mathrm{g})$, gentamicin (GM) $(10 \mu \mathrm{g})$, ciprofloxacin (CIP) $(5 \mu \mathrm{g})$, ofloxacin (OFX) $(10 \mu \mathrm{g})$, imipenem (IMI) $(10 \mu \mathrm{g})$, meropenem (MEM) $(10 \mu \mathrm{g})$, and colistin sulphate (CO) $(10 \mu \mathrm{g})$ (Mast diagnostics, Merseyside, U.K.). K. pneumoniae was considered MDR if an isolate was found resistant to three or more antibiotics. ESBLs were studied according to CLSI guidelines ${ }^{11}$ using inhibitor-potentiated diffusion test with ceftazidime and cefotaxime, each disk with and without clavulanic acid.

\section{Modified Hodge test}

The test was performed according to the CLSI instructions. Briefly, a bacterial suspension equivalent to $0.5 \mathrm{McF}$ arland was prepared with E. coli ATCC 25922 strain, and then diluted 1:10 using sterile saline solution. The diluted suspension was inoculated onto Mueller- Hinton agar plate using sterile swab by performing lawn culture. To dry the inoculum, plate was incubated at $37^{\circ} \mathrm{C}$ for three to five minutes. A $10 \mu \mathrm{g}$ meropenem disk was placed in the center of plate. The tested isolate was then streaked in a straight line from the edge of the disk to the edge of the plate. This was done carefully to prevent the swab from touching the disk. The plate was incubated at $37^{\circ} \mathrm{C} \pm 2$ for 16 to 24 hours. After the incubation, the susceptible zone in the clover leaf-type indentation in the carbapenemase producing samples was examined. The shape of sensitive zone of the positive control sample was compared to the tested samples to determine the positive cases in the Hodge test. In each plate, depending on its size, two to four samples were cultured in a straight line to perform the Hodge Test. ${ }^{11}$

\section{Minimum inhibitory concentration determination}

For imipenem and meropenem Minimum Inhibitory Concentration (MIC) determination, E-test strips were used following the manufacturer's instructions. A bacterial suspension with turbidity-adjusted equivalent to $0.5 \mathrm{McF}$ arland standard was prepared from a $16-18 \mathrm{~h}$ culture from blood agar plate. The suspension was inoculated onto Mueller-Hinton Agar plate. The E-test strip was placed on the surface of agar that has been inoculated with a lawn of test bacteria using sterile forceps and then plate was incubated under suitable conditions as outlined above for Kirby-Bauer method. The MIC was interpreted directly from the graduated E-test strip where bacterial growth of inhibition showed an ellipse around the strip. The results were interpreted according to the CLSI breakpoints. ${ }^{11}$

\section{DNA extraction and detection of bla $_{K P C 2}$ gene}

DNA was extracted from K. pneumoni$a e$ isolates using the commercial DNA extraction kit (Stratec Biomedical systems, Birkenfeld, Germany). Briefly, a bacterial suspension matched equivalent to 0.5 McFarland was prepared from an overnight culture and then centrifuged. DNA was extracted as per the instructions provided with the kit from the pellet and finally resolved in $100 \mu 1$ TE buffer. ${ }^{12}$

For PCR, primers producing $310 \mathrm{bp}$ amplified fragment $\left(b l a_{\mathrm{KPC} 2}-F \quad 5^{\prime}\right.$ ' GGCAGCCGTGCAATAC-3'; bla $\mathrm{KPC2}_{2}-\mathrm{R}$ 5'GCCGCATAACTCGTTCA-3') were used. ${ }^{13}$ DNA amplifications were performed in 20 $\mu 1$ volumes that contained 10 to $100 \mathrm{ng}$ of DNA, $0.5 \mu \mathrm{M}$ of each primer, in the presence of $2 \mathrm{mM} \mathrm{MgCl}_{2}, 100 \mu \mathrm{M}$ of each dNTP, 10X PCR buffer and 2.5U recombinant DNA polymerase (Cinnagen, Iran). Amplification was performed in a DNA thermal cycler (Gradient Eppendorf) programmed at $94^{\circ} \mathrm{C}$ ( $6 \mathrm{~min}$ ) for initial denaturation step followed by 35 cycles: each at $94^{\circ} \mathrm{C}(50 \mathrm{~s}), 57^{\circ} \mathrm{C}(50 \mathrm{~s})$, and $72^{\circ} \mathrm{C}(55 \mathrm{~s})$, and final extension step at $72^{\circ} \mathrm{C}$ for 7 minutes. Gel electrophoresis was performed for 45 $\mathrm{min}$ in a $1.2 \%$ agarose gel at $80 \mathrm{~V}$ and staining performed with SYBR Safe - DNA Gel Stain to visualize gel under UV light. Determination size of fragments was comparing with 100 bp DNA ladder size marker (Pishgam,Iran). ${ }^{12}$

\section{Statistical method}

The results were analyzed using descriptive statistics in SPSS software for Windows (version 19 SPSS Inc., Chicago, IL, USA).

\section{Results}

A total $104 K$. pneumoniae isolates were collected during 2016-2017 from 60 (57.7\%) male and 44 (42.3\%) female patients admitted to various wards of a University teaching hospital, Tabriz, Iran.

According to the disk agar diffusion test, the highest frequency of resistance was found to co-trimaxazole $(69.2 \%)$, followed by ceftazidime $(66.3 \%)$, ceftriaxone $(65.4 \%)$, ofloxacin and ciprofloxacin $(54.8 \%)$, gentamicin $(50 \%)$, and amikacin (39.4\%). The frequency of imipenem and meropenem resistance was $31.7 \%$ and $32.7 \%$, respectively. The most effective antibiotic was colistin with its susceptibility rate being $98.1 \%$. Among 104 K. pneumoni$a e, 24$ isolates were found MDR, making the prevalence as $11.5 \%$. Of all $K$. pneumoniae enrolled in the study, 69 (66.3\%) isolates were found resistant to 3rd-generation cephalosporins and among them 38 (55.1\%) were ESBL producers using the inhibitor potentiated disk method.

Resistance to carbapenems was initially screened based on the resistance to meropenem or imipenem and 38 (36.5\%) isolates were found carbapenem resistant. Among these isolates, 29 isolates were resistant to both imipenem and meropenem, 4 isolates were resistant to imipenem only and 5 isolates showed resistance to meropenem only.

Among 38 carbapenem resistant isolates, $27(71.1 \%)$ isolates had imipenem MIC higher than resistance criteria documented by CLSI and 3 isolates $(7.9 \%)$ were found intermediate resistant. MIC range, $\mathrm{MIC}_{50}$ and $\mathrm{MIC}_{90}$ were $0.19-32 \mu \mathrm{g} / \mathrm{ml}$, $4 \mu \mathrm{g} / \mathrm{ml}$ and $16 \mu \mathrm{g} / \mathrm{ml}$, respectively.

Modified Hodge test (MHT) was positive for $24(63.1 \%)$ isolates while, bla $_{K P C-2}$ gene was detected in $8(21.05 \%)$ isolates. These eight isolates were positive for MHT. Imipenem MIC range was 4-32 $\mu \mathrm{g} / \mathrm{ml}$ for $b l a_{K P C-2}$ positive isolates. All of $b l a_{K P C-2}$ positive isolates were colistin susceptible and co-trimaxazole and ceftazidime resistant. The antimicrobial susceptibility and clinical feature of $b l a_{K P C-2}$ positive isolates are presented in Table 1.

\section{Discussion}

$K$. pneumoniae has evolved as the most common pathogens in healthcare settings due its high levels of resistance to antibiotics. ${ }^{14}$ In the present study, antibiotic susceptibility assay presented variable frequency of resistance to the tested antibiotics. Similar to other studies, most of our isolates were resistant towards co-trimoxazole, cephalosporins, fluoroquinolones and aminoglycosides. One of the main problems associated with Enterobacteriaceae is the distribution of production of ESBLs, the enzymes which convert all $\beta$-lactam antibiotics drugs to inactive form except cephamycins and carbapenems. ${ }^{14,15}$ The high frequency of resistance to ceftazidime $(66.3 \%)$ and ceftriaxone $(65.4 \%)$ found in the present study indicates inefficiency of these drugs for empirical therapy in our settings. The genes encoding ESBLs are commonly plasmid-mediated, and additional resistance elements to others class of antibiotics such as aminoglycosides, fluoroquinolones are often co-located on the same 
Table 1. Antibiotic resistance pattern in the Klebsiella pneumoniae carbapenemase producing $K$. pneumoniae.

\begin{tabular}{|c|c|c|c|c|c|c|c|c|c|c|c|c|c|c|c|}
\hline \multirow[t]{2}{*}{ Gender } & \multirow[t]{2}{*}{ Age } & \multirow[t]{2}{*}{ Source } & \multirow[t]{2}{*}{ MHT } & \multicolumn{2}{|c|}{$\mathrm{MIC}(\mu \mathrm{g} / \mathrm{ml})$} & \multicolumn{10}{|c|}{ Antibiotics (Disk diffusion assay) } \\
\hline & & & & IMI & MEM & IMI & MRO & CAZ & CRO & GEN & AMK & CIP & OFX & TS & $\mathrm{CO}$ \\
\hline M & 39 & Urine & + & 4 & 4 & $\mathrm{R}$ & $\mathrm{R}$ & $\mathrm{R}$ & $\mathrm{R}$ & $\mathrm{R}$ & S & $\mathrm{R}$ & S & $\mathrm{R}$ & S \\
\hline M & 65 & Wound & + & 4 & 32 & $\mathrm{R}$ & $\mathrm{R}$ & $\mathrm{R}$ & S & S & S & $\mathrm{R}$ & $\mathrm{R}$ & S & S \\
\hline M & 48 & Wound & + & 8 & 6 & $\mathrm{R}$ & $\mathrm{R}$ & $\mathrm{R}$ & $\mathrm{R}$ & $\mathrm{R}$ & S & $\mathrm{R}$ & $\mathrm{R}$ & $\mathrm{R}$ & S \\
\hline $\mathrm{F}$ & 12 & Urine & + & 4 & 1 & $\mathrm{R}$ & S & $\mathrm{R}$ & $\mathrm{R}$ & $\mathrm{R}$ & S & $\mathrm{R}$ & $\mathrm{R}$ & $\mathrm{R}$ & S \\
\hline M & 50 & Wound & + & 16 & 8 & $\mathrm{R}$ & $\mathrm{R}$ & $\mathrm{R}$ & S & $\mathrm{R}$ & S & $\mathrm{R}$ & S & $\mathrm{R}$ & S \\
\hline M & 9 & Blood & + & 4 & 0.5 & $\mathrm{R}$ & S & $\mathrm{R}$ & $\mathrm{R}$ & $\mathrm{R}$ & $\mathrm{R}$ & $\mathrm{R}$ & $\mathrm{R}$ & $\mathrm{R}$ & S \\
\hline M & 47 & Respiratory & + & 32 & 8 & $\mathrm{R}$ & $\mathrm{R}$ & $\mathrm{R}$ & $\mathrm{R}$ & $\mathrm{R}$ & $\mathrm{R}$ & $\mathrm{R}$ & $\mathrm{R}$ & $\mathrm{R}$ & S \\
\hline $\mathrm{F}$ & 46 & Urine & + & 8 & 4 & $\mathrm{R}$ & $\mathrm{R}$ & $\mathrm{R}$ & $\mathrm{R}$ & $\mathrm{R}$ & S & S & $\mathrm{R}$ & $\mathrm{R}$ & S \\
\hline
\end{tabular}

AMK: amikacin; CAZ: ceftazidime; CIP: ciprofloxacin; CO: colistin; TS: co-trimoxazole; CRO: ceftriaxone; GEN: gentamicin; IMI: imipenem; OFX: ofloxacin; MEM: meropenem. F: Female; M: Male; R: Resistant; S: Sensitive; MHT: Modified Hodge test; MIC: Minimum Inhibitory Concentration.

plasmid. Therefore, many of the ESBLs producing isolates express cross-resistance between antimicrobial agents of different class and are multidrug resistant. Therefore, they are identified as a considerable therapeutic limitation for both clinicians and clinical laboratory. ${ }^{15-17}$ Carbapenems have been the major last resort of choice for the treatment of infections caused by these MDR isolates. ${ }^{17}$ However, frequent administration of carbapenems has led to carbapenem resistant $K$. pneumoniae. $^{14}$ Different researchers have reported the different frequency of resistance to carbapenem in Enterobacteriaceae. Yan et al. reported 5.8\% carbapenem resistant among clinical isolates of Enterobacteriaceae. ${ }^{18}$ Japoni et al., in 2014 , reported $12 \%$ carbapenem resistance in K. pneumoniae from Iran. ${ }^{19}$ Duin et al. tracked molecular epidemiology of carbapenem resistant $K$. pneumoniae infection and reported carbapenem resistance in the organism isolated from $45 \%$ of elderly long-term care population. ${ }^{20}$ In the present study $25.9 \%$ of $K$. pneumoniae isolates were carbapenem resistant by MIC determination method. These discrepancies in frequency of carbapenem resistant $K$. pneumoniae may be due to geographical differences, patterns of antibiotic use and the population selected in different studies. Admission to the ICU, a central venous catheter, exposure to antibiotics and diabetes mellitus have been proposed as the risk factors for carbapenem resistance in $K$. pneumoniae $^{21}$ Colistin is one of the last remaining choices effective for treatment of invasive infections due to carbapenem resistant $K$. pneumoniae. In the present study, colistin was found the most effective drug against $K$. pneumoniae isolates with its susceptibility rate being $100 \%$. On the contrary there have been reports of colistin resistant, carbapenem resistant Enterobacteriaceae from different regions of the world. ${ }^{6}$ In the United States, car- bapenem-resistant $K$. pneumoniae constitutes $92 \%$ of all carbapenem-resistant Enterobacteriaceae and carbapenemase production mediated by $b l a_{\mathrm{KPC}}$ is the most prevalent mechanism conferring resistance to carbapenems. ${ }^{7}$ A three year period study from China showed $b l a_{K P C-2}$ gene as the major predominant $(65.0 \%)$ carbapenemase among $K$. pneumoniae. ${ }^{22}$ On the other hand, some others studies did not detect $b l a_{K P C-2}$ gene by the PCR among carbapenemase producer $K$. pneumoniae strains..$^{23,24}$ This difference can be due to presence of diverse types of resistance or porins deficiency. ${ }^{23,24}$ In addition, in view of the emerging carbapenem resistance, the performance of detection test is essential for accuracy of carbapenemase detection. For example, resistance to ertapenem disk is not definite reason for carbapenemase production, principally when carbapenemase production is infrequent. ${ }^{24}$ The KPC confers resistance to all $b$-lactam agents and have the significant potential for wide spread. Laboratory detection of bla $\mathrm{KPC}-2_{2}$ producing clinical isolates is thus, essential for controlling the spread of this resistance mechanism. ${ }^{25}$ In the present study, bla $_{K P C-2}$ gene were found in $8(21.1 \%)$ carbapenem resistant isolates that were positive for MHT too. All of these isolates were resistance to imipenem on the disk diffusion assay but meropenem resistance was detected in $6(75 \%)$ of bla $_{K P C-2}$ positive isolates.

Given the limited therapeutic options available, the accurate and timely detection carbapenemase-producing bacteria is vital in order to control their spread. The CLSI guidelines for Enterobacteriaceae base on meropenem and ertapenem as well as Modified Hodge test however, pros and cons of each test are still debatable. Imipenem disk diffusion assay has been reported as an applicable and easy method of detection carbapenemase-producing Enterobacteriaceae without the need for approval of carbapenemase production by performing the MHT as recommended by the CLSI. ${ }^{26}$

\section{Conclusions}

High frequency of carbapenem resistant K. pneumoniae was observed in our hospital setting and bla $_{K P C-2}$ as a common mechanism. Our finding was shown imipenem disk diffusion is applicable and quick methods for detection of these isolates. Infection management and antibiotics stewardship interferences to control the spread of these pathogens and their related resistance towards conventional antibiotics are required.

\section{References}

1. Edwards JR, Peterson KD, Andrus ML, et al. National Healthcare Safety Network (NHSN) report, data summary for 2006 through 2007, issued November 2008. Am J Infect Control 2008;36:609-26.

2. Michalopoulos A, Virtzili S, Rafailidis $\mathrm{P}$, et al. Intravenous fosfomycin for the treatment of nosocomial infections caused by carbapenem-resistant Klebsiella pneumoniae in critically ill patients: a prospective evaluation. Clin Microbiol Infect 2010;16:184-6.

3. Snitkin ES, Zelazny AM, Thomas PJ, et al. Tracking a hospital outbreak of carbapenem-resistant Klebsiella pneumoniae with whole-genome sequencing. Sci Transl Med 2012;4:148.

4. Cuzon G, Ouanich J, Gondret R, et al. Outbreak of OXA-48-positive carbapenem-resistant Klebsiella pneumoniae isolates in France. Antimicrob Agents Chemother 2011;55:2420-3.

5. Schwaber MJ, Klarfeld-Lidji S, Navon- 
Venezia S, et al. Predictors of carbapenem-resistant Klebsiella pneumoniae acquisition among hospitalized adults and effect of acquisition on mortality. Antimicrob Agents Chemother 2008;52:1028-33.

6. Marchaim D, Chopra T, Pogue JM, et al. Outbreak of colistin-resistant, carbapenem-resistant Klebsiella pneumoniae in metropolitan Detroit, Michigan. Antimicrob Agents Chemother 2011;55:593-9.

7. Lledo W, Hernandez M, Lopez E, et al. Guidance for control of infections with carbapenem-resistant or carbapenemase-producing Enterobacteriaceae in acute care facilities. Morbidity Mortal Weekly Repo 2009;58:256-8.

8. Kang C-I, Song J-H. Antimicrobial resistance in Asia: current epidemiology and clinical implications. Infect Chemother 2013;45:22-31.

9. Campos AC, Albiero J, Ecker AB, et al. Outbreak of Klebsiella Pneumoniae carbapenemase-producing $\mathrm{K}$. pneumoniae: A systematic review. Am J Infect Control 2016;44:1374-80.

10. Tumbarello M, Viale P, Viscoli C, et al. Predictors of mortality in bloodstream infections caused by Klebsiella pneumoniae carbapenemase-producing $\mathrm{K}$. pneumoniae: importance of combination therapy. Clin Infect Dis 2012;55:943-50.

11. Patel J, Cockerill F, Alder J, et al. Performance standards for antimicrobial susceptibility testing; twenty-fourth informational supplement. CLSI Standards for Antimicrobial Susceptibility Testing 2014;34:1-226.

12. Asgharzadeh M, Kafil HS, Khakpour M. Comparison of mycobacterial interspersed repetitive unit-variable number tandem repeat and IS6110-RFLP meth- ods in identifying epidemiological links in patients with tuberculosis in Northwest of Iran. Ann Microbiol 2008;58:333-9.

13. Sadeghi J, Mansouri S. Molecular characterization and antibiotic resistance of clinical isolates of methicillin-resistant Staphylococcus aureus obtained from Southeast of Iran (Kerman). Apmis 2014;122:405-11.

14. Sun K, Chen X, Li C, et al. Clonal dissemination of multilocus sequence type 11 Klebsiella pneumoniae carbapenemase-producing $K$. pneumoniae in a Chinese teaching hospital. Apmis 2015;123:123-7.

15. El Bouamri M, Arsalane L, El Kamouni Y, Zouhair S. Antimicrobial susceptibility of urinary Klebsiella pneumoniae and the emergence of carbapenemresistant strains: A retrospective study from a university hospital in Morocco, North Africa. Afr J Urol 2015;21:36-40.

16. Cantón R, Coque TM. The CTX-M $\beta$ lactamase pandemic. Curr Opin Microbiol 2006;9:466-75.

17. Wang J-T, Wu U-I, Lauderdale T-LY, et al. Carbapenem-nonsusceptible Enterobacteriaceae in taiwan. PloS One 2015;10:e0121668.

18. Yan J, Lee N, Chen $H$, et al. Bloodstream infections caused by IMP8-producing Enterobacteriaceae isolates: the need for clinical laboratory detection of metallo- $\beta$-lactamases? Eur $\mathrm{J}$ Clin Microbiol Infect Dis 2013;32:345-52.

19. Japoni-Nejad A, Ghaznavi-Rad E, van Belkum A. Characterization of plasmidmediated AmpC and carbapenemases among Iranain nosocomial isolates of Klebsiella pneumoniae using phenotyping and genotyping methods. Osong Public Health Res Perspect 2014;5:333-
8.

20. Van Duin D, Perez F, Rudin SD, et al. Surveillance of carbapenem-resistant Klebsiella pneumoniae: tracking molecular epidemiology and outcomes through a regional network. Antimicrob Agents Chemother 2014;58:4035-41.

21. Giannella M, Trecarichi EM, De Rosa $F$, et al. Risk factors for carbapenem-resistant Klebsiella pneumoniae bloodstream infection among rectal carriers: a prospective observational multicentre study. Clin Microbiol Infect 2014;20:1357-62.

22. Cheng L, Cao X-L, Zhang Z-F, et al. Clonal dissemination of KPC-2 producing Klebsiella pneumoniae ST11 clone with high prevalence of oqxAB and rmtB in a tertiary hospital in China: results from a 3-year period. Ann Clin Microbiol Antimicrob 2016;15:1.

23. Bina M, Pournajaf A, Mirkalantari S, et al. Detection of the Klebsiella pneumoniae carbapenemase (KPC) in K. pneumoniae Isolated from the Clinical Samples by the Phenotypic and Genotypic Methods. Iran J Pathol 2015;10:199.

24. Flonta M, Lupse M, Craciunas C, et al. Ertapenem resistance among extendedspectrum- $\beta$-lactamase producing Klebsiella pneumoniae isolates. Therap Pharmacol Clin Toxicol 2011;15:121-5.

25. Anderson K, Lonsway D, Rasheed J, et al. Evaluation of methods to identify the Klebsiella pneumoniae carbapenemase in Enterobacteriaceae. J Clin Microbiol 2007;45:2723-5.

26. Benenson S, Temper V, Cohen MJ, et al. Imipenem disc for detection of KPC c a r b a pen e m a s e - producing Enterobacteriaceae in clinical practice. J Clin Microbiol 2011;49:1617-20. 